\title{
AlAs 2D electrons in an antidot lattice: Electron pinball with elliptical Fermi contours
}

\author{
O. Gunawan, E. P. De Poortere and M. Shayegan \\ Department of Electrical Engineering, Princeton University, Princeton, NJ 08544
}

(Dated: November 16, 2018)

\begin{abstract}
We report ballistic transport measurements in a two-dimensional electron system confined to an AlAs quantum well and patterned with square antidot lattices of period $a=0.6,0.8,1.0$ and $1.5 \mu \mathrm{m}$. In this system two in-plane conduction-band valleys with elliptical Fermi contours are occupied. The low-field magneto-resistance traces exhibit peaks corresponding to the commensurability of the cyclotron orbits and the antidot lattice. From the dependence of the position of the peak associated with the smallest commensurate orbit on electron density and $a$, we deduce the ratio of the longitudinal and transverse effective masses $m_{l} / m_{t}=5.2 \pm 0.4$, a fundamental parameter for the anisotropic conduction bands in AlAs.

PACS numbers: 72.20.-i, 73.23.Ad, 75.47.Jn
\end{abstract}

The crystal structure and lattice constant of AlAs are closely matched to that of GaAs and, as a result, AlAs is widely used as a barrier material in AlAs/GaAs heterostructures. However, AlAs differs greatly from GaAs in its band-structure. In contrast to GaAs where electrons occupy a single, isotropic, conduction-band minimum at the center of the Brillouin zone, electrons in AlAs populate anisotropic conduction-band minima (valleys) centered at the X-points of the Brillouin zone. The constant energy surface in (bulk) AlAs consists of six, anisotropic, half-ellipsoids (three full-ellipsoids). It is also possible to confine electrons to a modulation-doped AlAs quantum well, thereby forming a high-mobility twodimensional electron system (2DES) $\underline{\underline{1}}$ If the quantum well is wider than about $5 \mathrm{~nm}$, the electrons occupy only the two conduction-band valleys with their major axes in the plane, along the [100] and [010] crystal axes. We refer to these two valleys as $X$ and $Y$, respectively [Fig. 1(b)]. Here we report ballistic transport measurements in a square anti-dot (AD) array ${ }^{2,3,4}$ patterned in a high-mobility AlAs 2DES. The data provide a direct measure of the anisotropy of the Fermi contour, or equivalently, the ratio of the longitudinal and transverse electron effective masses, a fundamental parameter of the AlAs conduction-band. Recently it has been shown that this anisotropy can be exploited to realize a simple "valley-filter" device using a quantum point contact structure.$\underline{\underline{5}}$ Such a device may play an important role in "valleytronics" or valley-based electronics applications, $\underline{6}$ or for quantum computation where the valley state of an electron might be utilized as a qubit. $\underline{\underline{7}}$

We performed experiments on 2DESs confined to a high-quality, modulation-doped, $15 \mathrm{~nm}$-wide, AlAs quantum well grown by molecular beam epitaxy on a (001) GaAs substrate. ${ }^{\underline{1}}$ The AlAs quantum well is flanked by undoped layers of $\mathrm{Al}_{0.4} \mathrm{Ga}_{0.6} \mathrm{As}$ on each side, and the doping is via a delta-layer of $\mathrm{Si}$ on the surface side at a distance of $100 \mathrm{~nm}$ from the well and $32 \mathrm{~nm}$ from the surface. We patterned a Hall bar sample, with the current direction along [100], using standard optical photolithography. We then deposited a layer of polymethyl methacrylate (PMMA) and patterned the AD arrays us-
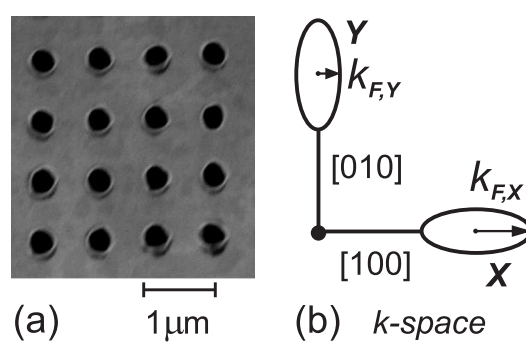

(b) $k$-space

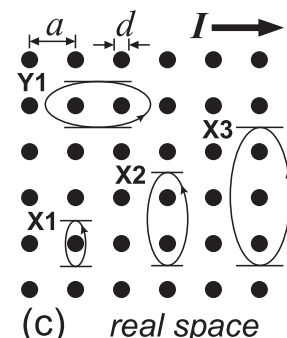

FIG. 1: (a) Micrograph of the AD lattice region with period $a=0.8 \mu \mathrm{m}$. (b) The Fermi contours of AlAs in-plane valleys $X$ and $Y$ in $k$-space. The Fermi wave vectors $k_{F, X}$ and $k_{F, Y}$ are indicated. (c) The first four commensurate orbits for the $X$ and $Y$ valleys that give rise to peaks in magneto-resistance for current along the $x$-direction; these orbits have diameters in the $y$-direction that are equal to a multiple integer of the AD lattice period (see text).

ing electron beam lithography. The PMMA layer served as a resist for a subsequent dry etching process used to define the $\mathrm{AD}$ holes. We used an electron cyclotron resonance etching system with an $\mathrm{Ar} / \mathrm{Cl}_{2}$ plasma, $\stackrel{\underline{8}}{\text { at }}$ an etch rate of $\simeq 55 \mathrm{~nm} / \mathrm{min}$, to obtain small feature sizes without a degradation of the $2 \mathrm{DES}$ quality. The $\mathrm{AD}$ pattern was etched to a depth of $\simeq 80 \mathrm{~nm}$, thus stripping the dopant layer and depleting the electrons in the $\mathrm{AD}$ regions. The micrograph of a section of one of our AD arrays is shown in Fig. 1(a). Each AD array is a square lattice and covers a $20 \mu \mathrm{m} \times 30 \mu \mathrm{m}$ area. There are four regions of $\mathrm{AD}$ lattice with different periods: $a=0.6,0.8,1.0$, and 1.5 $\mu \mathrm{m}$ as schematically shown in the inset of Fig. 2, The aspect ratio $d / a$ of each $\mathrm{AD}$ cell is $\sim 1: 3$, where $d$ is the $\mathrm{AD}$ diameter. Finally, we deposited a front gate, covering the entire surface of the active regions of the sample to control the 2DES density. Following an initial back-gate biasing and brief illumination,,$\frac{9}{-}$ we used the front gate to tune the total density $\left(n_{T}\right)$ from 2 to $5 \times 10^{11} / \mathrm{cm}^{2}$. The density was determined from both Shubnikov-de Haas oscillations and Hall coefficient measurements that agree with each other. From the measurements on an unpatterned Hall bar region in a different sample but from the 


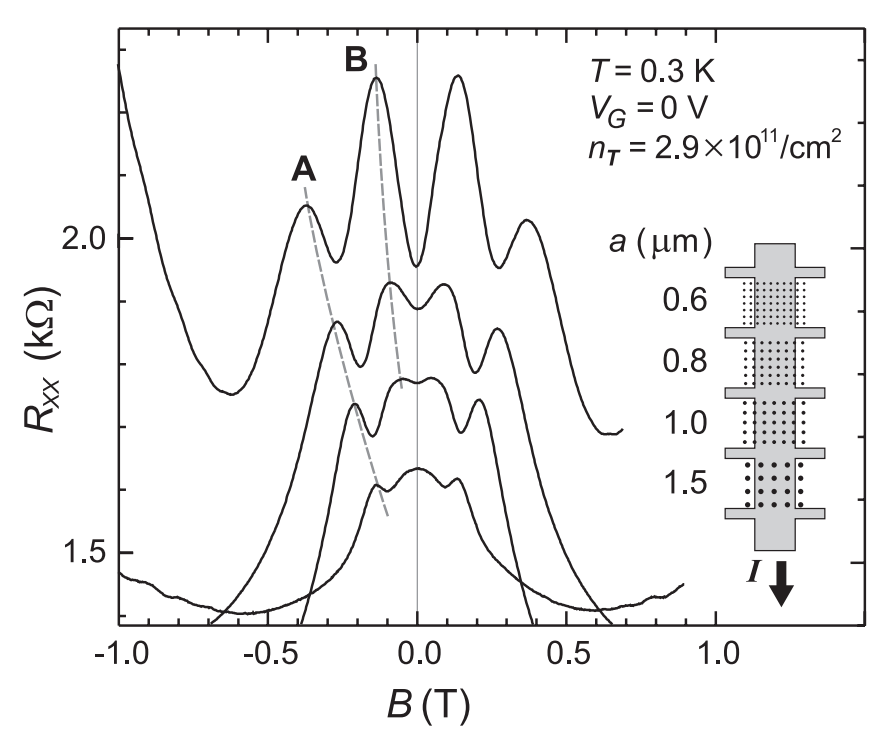

FIG. 2: Low-field MR traces for all four $\mathrm{AD}$ regions with periods (from top to bottom) equal to $a=0.6,0.8,1.0$, and $1.5 \mu \mathrm{m}$. Traces are offset vertically for clarity, except for the bottom one. The Hall bar with the different AD regions is schematically shown on the right.

same wafer, we obtain a mobility of $\sim 10 \mathrm{~m}^{2} /$ Vs at a typical density of $3 \times 10^{11} / \mathrm{cm}^{2}$ and $T=0.3 \mathrm{~K}$. This gives a typical mean-free-path of $\sim 1 \mu \mathrm{m}$.

Figures 2 and 3 summarize our main experimental results. Figure 2 shows the low-field magneto-resistance $(\mathrm{MR})$ traces, measured as a function of perpendicular magnetic field $(B)$, for all the $\mathrm{AD}$ regions. We observe two peaks, $\mathrm{A}$ and $\mathrm{B}$, which are symmetric with respect to $B=0$. Peak A, whose position is higher in field than peak $\mathrm{B}$, is seen in the traces from all the $\mathrm{AD}$ regions. Peak $\mathrm{B}$, on the other hand, is not observed in the $a=1.5$ $\mu \mathrm{m}$ trace. In general, we observe that, as the $\mathrm{AD}$ lattice period becomes smaller, the positions of both peaks A and $\mathrm{B}$ shift to higher field values (as indicated by the dashed lines). Figure 3 captures the gate-voltage $\left(V_{G}\right)$ dependence of the MR traces for the $a=0.8 \mu \mathrm{m} \mathrm{AD} \mathrm{re-}$ gion. As we increase $V_{G}$ to increase the 2DES density, peak A shifts to higher field values while peak B does not appear to shift. We have made similar observations in the other $\mathrm{AD}$ regions as $V_{G}$ is varied.

In order to analyze the data of Figs. 2 and 3 , we first briefly review what is known about ballistic transport in $\mathrm{AD}$ arrays for GaAs 2DESs where the Fermi contour is isotropic. Low-field MR traces for such systems typically exhibit commensurability peaks at magnetic fields where the classical cyclotron orbit fits around a group of ADs $, 2,3,4$ Although there are subtleties associated with the exact shape of the AD potential and also the possibility of chaotic orbits that bounce from one AD boundary to another, the peak observed at the highest magnetic field corresponds to the shortest period that fits around the smallest number of ADs. For an isotropic Fermi contour, this corresponds to a circular orbit, with

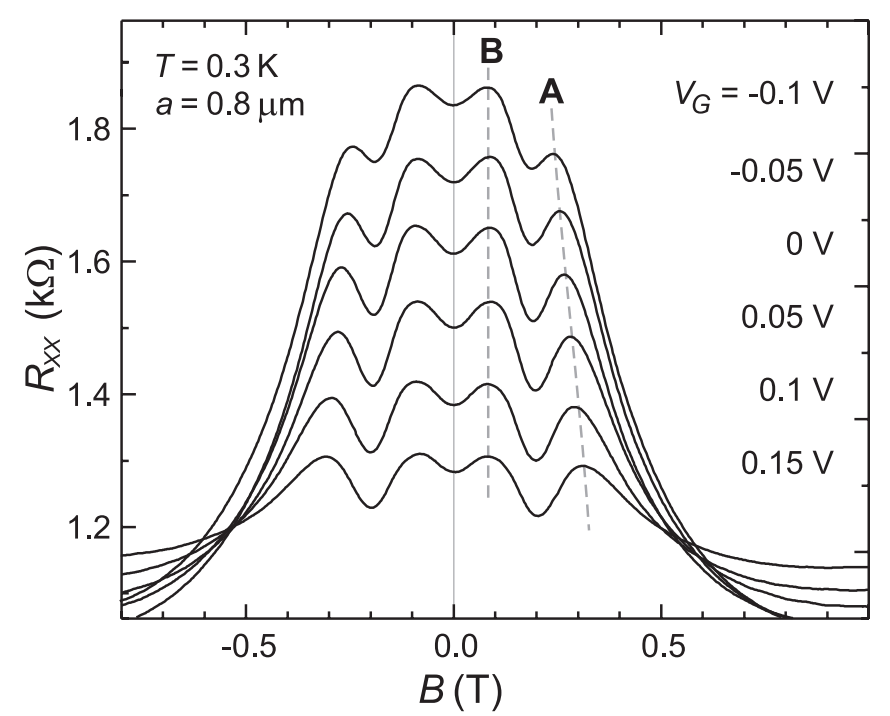

FIG. 3: Low-field MR traces for the AD region with period $a=0.8 \mu \mathrm{m}$ for $V_{G}=-0.1 \mathrm{~V}$ to $0.15 \mathrm{~V}$ (from top to bottom), corresponding to a linear variation of the density $n_{T}$ from 2.27 to $3.53 \times 10^{11} / \mathrm{cm}^{2}$. Traces are offset for clarity, except for the bottom one.

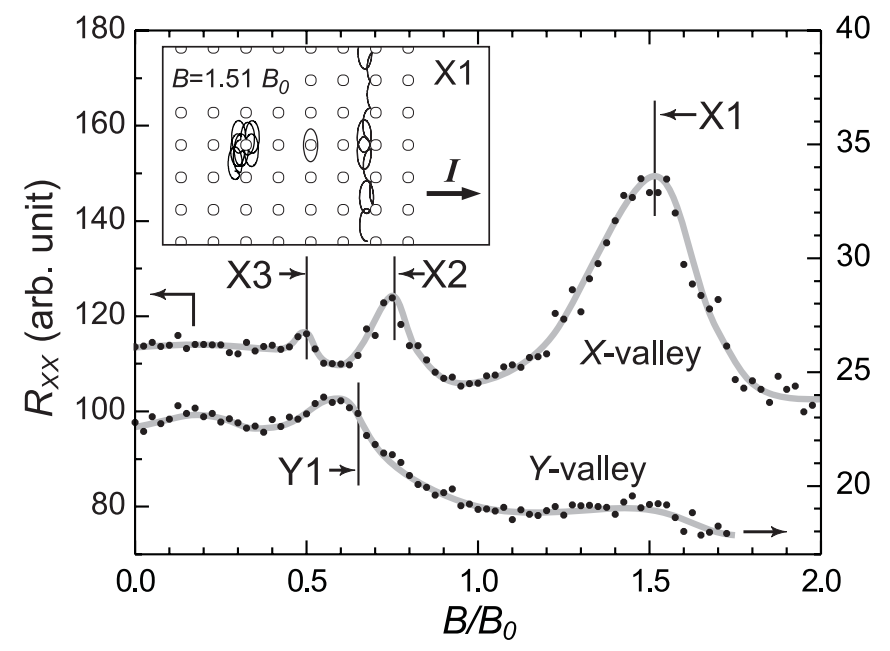

FIG. 4: MR obtained from numerical simulations (smooth curves are guides to the eye). Vertical lines indicate the expected positions of the peaks for orbits X1, X2, X3 and Y1, based on Fig. 1(c). We assumed equal densities for the two valleys and a current along the $x$-direction. $B_{0}$ is the magnetic field of the first commensurate orbit if the Fermi contour were circular. Inset: Simulation snapshot showing various types of trajectories: chaotic, pinned and skipping orbits, for $X$-valley electrons when X1 peak occurs $\left(B=\sqrt[4]{m_{l} / m_{t}} B_{0}\right)$.

a diameter equal to the $\mathrm{AD}$ period, encircling a single AD. There have also been studies of ballistic transport in 2DESs with isotropic orbits in an anisotropic (rectangular) $\mathrm{AD}$ lattice $\stackrel{10}{\underline{10}}$ Experimental results $\stackrel{11}{=}$ followed

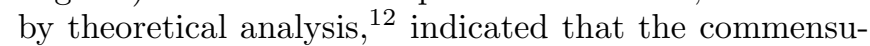
rability peaks are observed only when the orbit diam- 
eter matches an integer multiple of $\mathrm{AD}$ lattice period along the direction perpendicular to the current 13 Based on the above considerations, we can predict the first four (smallest) commensurate orbits of the $X$ and $Y$ valleys that may give rise to MR peaks in our system; these are shown in Fig. 1(c).

To evince this conjecture, we performed a kinematic, numerical simulation for our system, a 2DES with elliptical Fermi contours in an isotropic AD lattice in the presence of a perpendicular magnetic field $B$. The simulation details are similar to those described in Ref. 14 . The AD boundaries are represented as hard-wall potentials where the electrons are scattered elastically upon collision. A set of typical electron trajectories are shown in the inset of Fig. 4. Using the Kubo formula 14,15 we calculated the conductivity tensor and then obtained the resistance through its inversion. We performed the simulations for two separate cases, one for $X$ and one for $Y$-valley electrons and present the results in Fig. 4. The resulting MR traces indeed show peaks at or near the expected values, namely orbits X1, X2, X3 for the $X$-valley and $\mathrm{Y} 1$ for the $Y$-valley [Fig. 11(c)].

Now we focus our attention back on the experimental results. We associate peak A in our data of Figs. 2 and 3 with the shortest orbit X1 in Fig. 1(c). From the field position of this peak, and if we assume that the electron density of the $X$ valley is half the total density, we can directly obtain a value for the anisotropy (ratio of the major to minor axes diameters) of the elliptical orbits in our system, thus obtaining the effective mass ratio $m_{l} / m_{t}$. 16 However, there is a finite imbalance between the $X$ and $Y$ valley densities in our sample. Such imbalances can occur because of anisotropic strain in the plane of the sample and are very often present in AlAs 2DESs. 1,17 Therefore, we present here an analysis to determine the $m_{l} / m_{t}$ ratio independent of the density imbalance.

Consider a primary, commensurate orbit whose diameter in the direction perpendicular to the current is equal to the $\mathrm{AD}$ lattice period [orbits $\mathrm{X} 1$ and $\mathrm{Y} 1$ in Fig. 1(c)]. These would give rise to MR peaks at fields $B_{P}=2 \hbar k_{F} / e a$ where $k_{F}$ is the Fermi wavevector along the current direction. For the $X$ and $Y$ valleys, these wavevectors are $k_{F, X}$ and $k_{F, Y}$, respectively, as shown in Fig. 1(b). For an elliptical Fermi contour, they are related to the densities of the $X$ and $Y$ valleys, $n_{X}$ and $n_{Y}$, via the following relations:

$$
k_{F, X}^{2}=2 \pi n_{X} \sqrt{m_{l} / m_{t}}, \quad k_{F, Y}^{2}=2 \pi n_{Y} \sqrt{m_{t} / m_{l}} .
$$

Note that the total density $n_{T}=n_{X}+n_{Y}$ and the valley imbalance $\Delta n=n_{X}-n_{Y}$. We can obtain $n_{T}$ from the Shubnikov-de Haas oscillations of the MR at high magnetic fields or from a measurement of the Hall coefficient. Now consider orbit X1 as shown in Fig. 1(c). Its associated MR peak position $B_{P, X 1}$ is given as:

$$
B_{P, X 1}^{2}=\frac{h^{2}}{\pi e^{2} a^{2}} \sqrt{\frac{m_{l}}{m_{t}}}\left(n_{T}+\Delta n\right) .
$$

We use this expression to analyze our data.

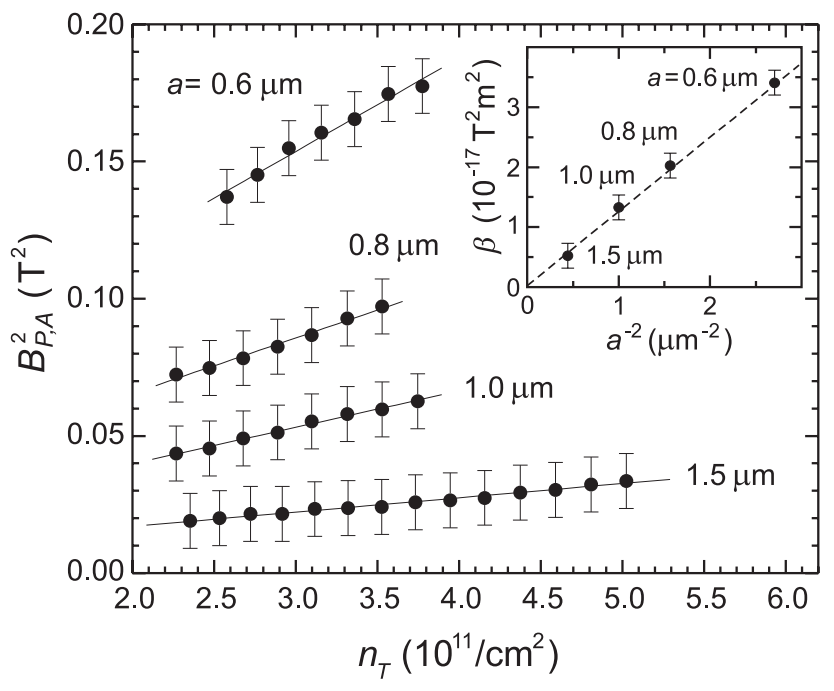

FIG. 5: Summary of the density dependence of $B_{P, A}^{2}$ for all four $\mathrm{AD}$ regions; $B_{P, A}$ is the position of peak $\mathrm{A}$ observed in MR traces. The straight lines are linear fits using Eq. 2 . The error bars reflect the uncertainty in the peak positions which were determined by subtracting second-order polynomial backgrounds from the MR traces. Inset: Slope $(\beta)$ of the $B_{P, A}^{2}$ vs $n_{T}$ lines of the main figure are plotted as a function of $a^{-2}$. The dashed-line is a linear fit to the data.

We assign peak $\mathrm{A}$ in our data to orbit X1 and plot the square of its field position $B_{P, A}^{2}$ as a function of $n_{T}$ in Fig. 5. It is clear that for all four AD lattice regions, $B_{P, A}^{2}$ varies linearly with $n_{T}$ as expected from Eq.2. Moreover, we obtain the slopes $(\beta)$ and the intercepts of the lines in Fig. [5] by performing a least-squares fit of each data set. Note that according to Eq. 2, $\beta=h^{2} \sqrt{m_{l} / m_{t}} / \pi e^{2} a^{2}$, and the intercept is equal to $\beta \Delta n \stackrel{18}{18}$ Finally, we plot $\beta$ as a function of $a^{-2}$ in the inset of Fig. 5. This figure shows that, consistent with the prediction of Eq. 2, $\beta$ indeed depends linearly on $a^{-2}$ and the line has a zero intercept.

From the slope, $\Delta \beta / \Delta\left(a^{-2}\right)$, of the line in Fig. [5] inset, we can deduce the effective mass anisotropy ratio:

$$
m_{l} / m_{t}=\pi^{2} e^{4} / h^{4}\left[\Delta \beta / \Delta\left(a^{-2}\right)\right]^{2} .
$$

Note that this mass anisotropy ratio is related to the slope of the line in Fig. 5 inset by a pre-factor containing only physical constants $\left(\pi^{2} e^{4} / h^{4}\right)$. Our data analysis and determination of this ratio are therefore insensitive to parameters such as density imbalance between the two valleys. From data of Fig. 5 we obtain $m_{l} / m_{t}=5.2 \pm 0.4$, in very good agreement with the ratio $m_{l} / m_{t}=5.2 \pm 0.5$ determined from recent ballistic transport measurements in AlAs 2DESs subjected to one-dimensional, periodic potential modulations ${ }^{17}$ Given the cyclotron mass in AlAs, $m^{*}=\sqrt{m_{l} m_{t}}=0.46 m_{0}, \frac{19}{1}$ we deduce $m_{l}=(1.05 \pm 0.1) m_{0}$ and $m_{t}=(0.20 \pm 0.02) m_{0}$, where $m_{0}$ is the free electron mass, in good agreement with the results of the majority of theoretical and experimental determinations of the effective mass in AlAs. 17 


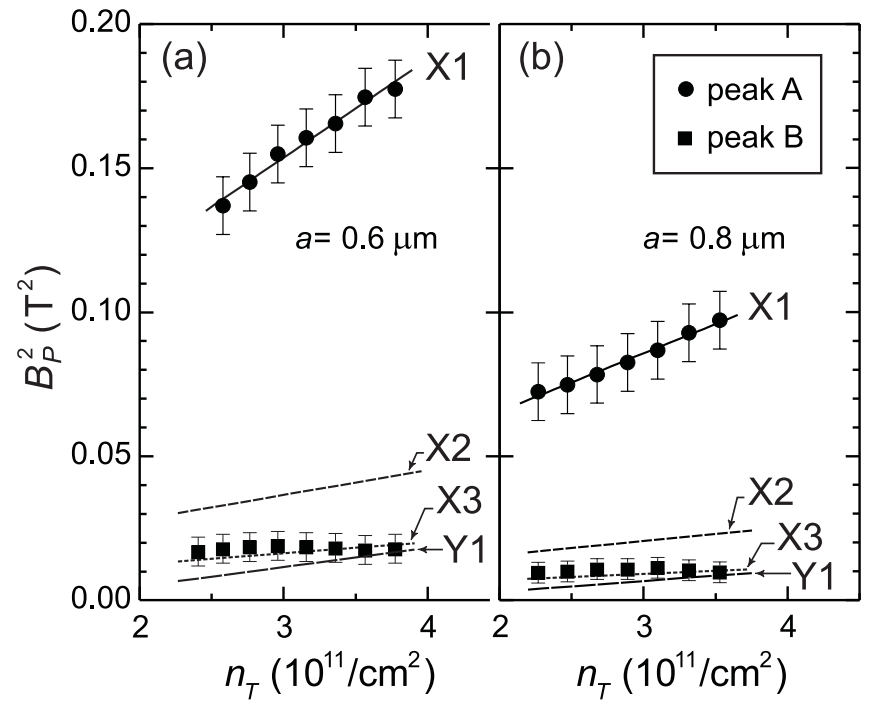

FIG. 6: Summary of the density dependence of $B_{P}^{2}$ of peak A and $\mathrm{B}$ for AD region: (a) $a=0.6 \mu \mathrm{m}$ and (b) $a=0.8 \mu \mathrm{m}$. The lines marked X1 are linear fits to peak A using Eq. 22. Lines $\mathrm{X} 2, \mathrm{X} 3$ and $\mathrm{Y} 1$ are the predicted peak positions calculated using equations similar to Eq. 2 (see text).

Several other features of the data presented here are noteworthy. From the intercepts of the linear fits in Fig. 5 we can determine the valley density imbalance for each AD lattice. Such analysis gives $\Delta n=1.5,1.2,1.0$, and $1.3 \times 10^{11} / \mathrm{cm}^{2}\left( \pm 0.2 \times 10^{11} / \mathrm{cm}^{2}\right)$ for the AD regions with $a=0.6,0.8,1.0$, and $1.5 \mu \mathrm{m}$, respectively. Such a variation of valley imbalance for different $\mathrm{AD}$ regions may come from non-uniform residual strain across the sample. Note that, because of the close proximity of the different $\mathrm{AD}$ lattice regions, we expect this variation to be small, consistent with the $\Delta n$ values deduced from the above analysis.

As for peak B, it is tempting to associate it with orbits
$\mathrm{X} 2$ or $\mathrm{Y} 1$ in Fig. 1(c). This is qualitatively consistent with the data of Fig. 2 which indicate that peak B moves to smaller values of magnetic field as the period of the AD lattice is made larger. Moreover, peak B becomes weaker with increasing AD lattice period and disappears for the largest period $a=1.5 \mu \mathrm{m}$. This is also consistent with the larger size of the X2 and Y1 orbits (compared to the $\mathrm{X} 1$ orbit), and the fact that for $a=1.5 \mu \mathrm{m}$, the lengths of these orbits become large compared to the electron meanfree-path. Quantitatively, using the values of $m_{l} / m_{t}$ and $\Delta n$ obtained above, we can modify Eq. 2 and determine the expected peak positions associated with the X2, X3 and Y1 orbits ${ }^{20}$ As illustrated in Fig. 6. we find that the predicted peaks for X2, X3 and Y1 orbits are close to each other in field and approximately straddle the observed positions of peak B in Fig. 2. ${ }^{21}$ It is possible then that peak B may originate from a superposition of X2, X3 and $\mathrm{Y} 1$ peaks that cannot be resolved in our experiment. We cannot rule out, however, that peak B may be strongly influenced by non-linear orbit resonances in the system. Such resonances are known to occur for orbits with long trajectories in the presence of a smooth $\mathrm{AD}$ potential. $\underline{\underline{4}}$

In conclusion, we performed ballistic transport experiments in AD lattices imposed on an AlAs 2DES where the electrons occupy two valleys with anisotropic Fermi contours. The low-field MR traces exhibit two sets of peaks. From the analysis of the positions of the peak associated with a commensurate orbit with the shortest trajectory [X1 orbit in Fig.11(c)], we deduced the effective mass anisotropy ratio $m_{l} / m_{t}$, a fundamental parameter of the AlAs conduction-band dispersion that cannot be directly measured from other transport experiments.

Our work was supported by the ARO, NSF, and the Alexander von Humboldt Foundation. We thank I. Trofimov and G. Sabouret for assistance with the electron cyclotron resonance etcher, and Y. P. Shkolnikov, K. Vakili, and R. Winkler for illuminating discussions.
1 E. P. De Poortere, Y. P. Shkolnikov, E. Tutuc, S. J. Papadakis, M. Shayegan, E. Palm, and T. Murphy, Appl. Phys. Lett. 80, 1583 (2002) and references therein.

2 D. Weiss, M. L. Roukes, A. Menschig, P. Grambow, K. von Klitzing, and G. Weimann, Phys. Rev. Lett. 66, 2790 (1991).

3 A. Lorke, J. P. Kotthaus, and K. Ploog, Phys. Rev. B. 44, 3447 (1991).

4 R. Fleischmann, T. Geisel, and R. Ketzmerick, Phys. Rev. Lett. 68, 1367 (1992).

5 O. Gunawan, B. Habib, E. P. De Poortere, and M. Shayegan, Phys. Rev. B 74, 155436 (2006).

6 A. Rycerz, J. Tworzydło, and C. W. J. Beenakker, condmat/0608533.

7 O. Gunawan, Y. P. Shkolnikov, K. Vakili, T. Gokmen, E. P. De Poortere, and M. Shayegan, Phys. Rev. Lett. 97, 186404 (2006).

${ }^{8}$ Y. W. Chen, B. S. Ooi, G. I. Ng, K. Radhakrishnan, and
C. L. Tan, J. Vac. Sci. Tech. B 18, 2509 (2000).

9 E. P. De Poortere, Y. P. Shkolnikov, and M. Shayegan, Phys. Rev. B 67, 153303 (2003).

10 The problem of ballistic transport for a 2DES with a circular Fermi contour in a rectangular AD lattice is equivalent to transport in a 2DES with an elliptical Fermi contour in a square $\mathrm{AD}$ lattice.

11 K. Tsukagoshi, S. Wakayama, K. Oto, S. Takaoka, K. Murase, and K. Gamo, Phys. Rev. B. 52, 8344 (1995).

12 W. Lu, Phys. Rev. B. 54, 8049 (1996).

13 This situation resembles the magnetic electron focusing effect in a system containing multiple, parallel onedimensional channels. See, e.g., K. Nakamura, D. C. Tsui, F. Nihey, H. Toyoshima, and T. Itoh, Appl. Phys. Lett. 56, 385 (1990) and F. Nihey, K. Nakamura, M. Kuzuhara, N. Samoto, and T. Itoh, Appl. Phys. Lett. 57, 1218 (1990).

14 T. Nagao, J. Phys. Soc. Jpn 64, 4097 (1995).

15 R. Kubo, J. Phys. Soc. Jpn. 12, 570 (1957). 
16 The ratio of the major to minor orbit diameter is $\sqrt{m_{l} / m_{t}}$.

17 O. Gunawan, Y. P. Shkolnikov, E. P. De Poortere, E. Tutuc, and M. Shayegan, Phys. Rev. Lett. 93, 246603 (2004).

18 We assume $\Delta n$ is fixed in the density range of interest.

19 T. S. Lay, J. J. Heremans, Y. W. Suen, M. B. Santos, K. Hirakawa, M. Shayegan, and A. Zrenner, Appl. Phys. Lett. 62, 3120 (1993).
20 To calculate the peak positions for orbit X2 and X3, simply replace $a$ with $2 a$ and $3 a$ in Eq. 2 For orbit Y1, replace $\sqrt{m_{l} / m_{t}}$ with $\sqrt{m_{t} / m_{l}}$ and $\Delta n$ with $-\Delta n$.

${ }^{21}$ In Fig. 6] the expected $B_{P}$ for Y1 orbits are lower than $B_{P}$ for X3 because $n_{Y}<n_{X}$ in our experiments. 